\title{
Unscented Kalman filter and Magnetic Angular Rate Update (MARU) for an improved Pedestrian Dead-Reckoning
}

\author{
Francisco Zampella*, Mohammed Khider ${ }^{\dagger}$, Patrick Robertson ${ }^{\dagger}$ and Antonio Jiménez* \\ ${ }^{*}$ Centre for Automation and Robotics (CAR), \\ Consejo Superior de Investigaciones Científicas (CSIC)-UPM \\ Ctra Campo Real km. 0.2, La Poveda, Madrid 28500, España \\ Email: francisco.zampella, antonio.jimenez@csic.es \\ ${ }^{\dagger}$ Institute for Communication and Navigation \\ German Aerospace Center (DLR) \\ Oberpfaffenhofen, Germany \\ Email: patrick.robertson, mohammed.khider@dlr.de
}

Web: http://www.car.upm-csic.es/lopsi

\begin{abstract}
The Extended Kalman Filter (EKF) has been the state of the art in Pedestrian Dead-Reckoning for foot-mounted Inertial Measurements Units. However due to the non-linearity in the propagation of the orientation the EKF is not the optimal Bayesian filter. We propose the usage of the Unscented Kalman Filter (UKF) as the integration algorithm for the inertial measurements. The UKF improves the mean and covariance propagation needed for the Kalman filter. Although the UKF provides a better estimate of the orientation, with Zero velocity UPdaTes (ZUPT) measurements, the yaw and the bias in the gyroscope associated with it becomes unobserved and might generate errors in the positioning. We studied the changes in the magnetic field during the stance phase and their relationship with the turn rates to propose three measurements using the magnetometer signal that will be called Magnetic Angular Rate Updates (MARUs). The first measurement uses the change in the angle of the magnetic field in the horizontal plane to measure the change in the yaw and provides a simple measurement for the UKF implementation. The second measurement relates the change in the magnetic field vector to the turn rate and provides information on the bias of the gyroscope for an UKF. The last measurement uses a first order approximation to generate a linear relationship with the gyroscope bias and therefore it can be used in an EKF. Finally we proposed a metric for the reliability of the stance as a way to use the pre and post stance information but adjusting the covariance of the measurements gradually from swing to stance. These methods were tested on real and simulated signals and they have shown improvements over the original PDR algorithms.
\end{abstract}

Index Terms-Pedestrian Dead Reckoning, Unscented Kalman Filter, Magnetically Aided Navigation, Soft Measurements.

\section{INTRODUCTION}

The recent improvements in the Micro Electro Mechanical (MEM) technology has allowed the development of Inertial Measurements Units (IMU) that due to thier size and weight can be easily carried by a pedestrian. Obtaining the position from a high grade IMU is done using an Inertial Navigation System (INS) [1], [2]. However high grade IMUs can not be carried by a pedestrian due to their size and weight. MEM IMUs present amounts of bias an noise that would generate large errors in few seconds. Many authors [3], [4], [5], [6], [7] had used the identification of the stance phase in a footmounted IMU to limit the growth of the errors. Among them Foxlin [4] was the first to use an Extended Kalman Filter
(EKF) to estimate and subtract the errors in the navigation states by imposing a Zero velocity UPdaTe (ZUPT) during the stance. Another common measurement as detailed in [5], is the assumption of a fixed orientation when the sensor is in a still phase, allowing a Zero Angular Rate Update (ZARU) that helps estimating the bias of the gyroscope. All these methods are based on a first order approximation of the propagation of a non-linear model of the states. We aim to propose a filter that is better adapted to the non-linear problem and to obtain information about the orientation from the magnetic measurements even in the presence of magnetic disturbances.

The use of a first order approximation of a non linear problem can lead to systematic errors, therefore we propose the use of an Unscented Kalman Filter (UKF) as stated in [8], [9]. Unlike the EKF, the UKF propagates the mean and covariance of the estimation using several sigma points around the estimated mean and then measures the mean and covariance of the propagated points. Theoretically the UKF is able to correctly estimate up to the third order term of the Taylor Series of the mean and up to the second order term of the Taylor series of the Covariance [10], while the EKF is capable of estimating only the first order term.

One of the mains sources of errors in PDR is the increment of the yaw error due to the fact that it is a non observable state. Many authors such as in [3], [11], [12] have used the Magnetic North measurements as direct measurements for the heading, but we have seen that due to the position related magnetic disturbances these measurements can generate errors in the position. In this work we propose to use the changes in the magnetic field during the stance to detect the turn rates of the sensor. This method can be used even in the presence of magnetic disturbances since they are position dependant. We will call this method Magnetic Angular Rate Update (MARU).

One of the main subjects in PDR is the Stance detection and many authors has proposed several detection methods [13], [14], [15], [15], [16]. They provide binary decisions that are usually optimized to avoid false stance detections by setting strong thresholds at the cost of reducing the stance samples. This reduces the amount of information that the filter receives and in some cases (fast walk, running, etc.) might result in 
not detecting the stances. In this work we propose the use of a soft threshold and a gradual change between the stance and swing phases as a way to implement a soft decision over the presence of a Stance.

This paper is structured as follows. In section II, we propose the structure of a general UKF for PDR estimation. In section III, we establish the concept of using the change in the magnetic field as source of information for the gyroscope and the orientation propagation. Three methods are proposed in this section to implement this measurement according to the available states and sensors. In section IV, we study the step detection and propose a method to gradually switch between swing and stance detection, providing an indication of the uncertainty of that measurement. In section $\mathrm{V}$, we evaluate the proposed methods with different test signals. In section VI, conclusions and future works are summarized.

\section{Unscented KALMAN FiLter}

Although the Extended Kalman Filter has proven to be a good estimator in PDR, under some conditions it might generate systematic errors due to the first order approximation of the orientation propagation. In this section, we will propose a new estimator; the Unscented Kalman Filter, which measures the evolution of the different states and the influence of the measurements using "sigma" points. The filter is usually separated into the Unscented Transformation (UT) that estimates the propagation of the states, and the filter update that associates the measurements with the changes in the states and correct them to minimize the covariance.

\section{A. Propagation of the states}

We will study first the evolution of the states, given measurements $A c c(k)=\left[A c c_{x}(k), A c c_{y}(k), A c c_{z}(k)\right]^{T}$ from the accelerometer and $\operatorname{Gyr}(k)=\left[\operatorname{Gyr}_{x}(k), \operatorname{Gyr}_{y}(k), \operatorname{Gyr}_{z}(k)\right]^{T}$ from the gyroscope in each sensor axis $(i=x, y, z)$. After the removal of the biases ( $\Delta a$ and $\Delta \omega$ respectively), we can obtain the turn rate $\omega_{b}(k)=\operatorname{Gyr}(k)-\Delta \omega(k)$ and the acceleration $a_{b}=A c c(k)-\Delta a$, both in the sensor reference frame (subindex $b$ ).

The measurements can be transformed to a navigation frame (subindex n, usually North-West-Up) using the Direction Cosine Matrix $C_{b}^{n}(k)$, so the velocity $\dot{r}(k)$ and position $r(k)$ can be obtained as:

$$
\begin{aligned}
& \dot{r}(k)=\dot{r}(k-1)+\left(C_{b}^{n}(k) \cdot a_{b}(k)-\vec{g}\right) \cdot \Delta t, \\
& r(k)=r(k-1)+\frac{(\dot{r}(k)+\dot{r}(k-1))}{2} \cdot \Delta t .
\end{aligned}
$$

The value of $C_{b}^{n}(k)$ is propagated using [1]:

$$
C_{b}^{n}(k)=C_{b}^{n}(k-1) \cdot e^{\Omega_{n b}^{b}(k) \cdot \Delta t},
$$

where the skew symmetrical matrix $\Omega_{n b}^{b}(k)$ is:

$$
\Omega_{n b}^{b}(k)=\left[\omega_{b}(k) \quad \times\right]=\left[\begin{array}{ccc}
0 & -\omega_{z}(k) & \omega_{y}(k) \\
\omega_{z}(k) & 0 & -\omega_{x}(k) \\
-\omega_{y}(k) & \omega_{x}(k) & 0
\end{array}\right],
$$

and is equivalent to the cross product with the vector $\omega_{b}(k)$. Equation 3 can be expressed as in [1]:

$$
e^{\Omega_{n b}^{b} \cdot \Delta t}=I+\frac{\sin (\|\omega \Delta T\|)}{\|\omega\|} \Omega_{n b}^{b}+\frac{(1-\cos (\|\omega \Delta T\|))}{\|\omega\|^{2}} \Omega_{n b}^{b}{ }^{2},
$$

and it represents the source of the non-linearities of the system. The state propagation is included in the function $x(k)=f(x(k-1), u(k))$, where $u(k)$ refers to the IMU measurements.

\section{B. Unscented Transformation}

The UKF measures the behavior of the transition with the Unscented Transformation. It uses several symmetrical "sigma" points around the mean of the states $\bar{x}_{k-1}$. The "sigma" points are established as in [10]:

$X_{k-1, i}=\left\{\begin{array}{ll}\bar{x}_{k-1} & i=0 \\ \bar{x}_{k-1}+\left(\sqrt{(n+\lambda) P_{k-1}}\right)_{i} & i=1, \ldots, n \\ \bar{x}_{k-1}-\left(\sqrt{(n+\lambda) P_{k-1}}\right)_{i-n} & i=n+1, \ldots, 2 n\end{array}\right.$,

where $\left(\sqrt{(n+\lambda) P_{k-1}}\right)_{i}$ represents the column i of the matrix squared root of the weighted covariance $P_{k-1}$ and it can be obtained using the eigenvalues decomposition. We will obtain $2 n+1$ sigma points, where $n$ is the number of states and $\lambda=\alpha^{2}(n+\kappa)-n$ is a scale factor where $\alpha$ and $\kappa$ are used to change the dispersion of the points.

Using a scaled squared root of the covariance, it is possible to obtain the covariance of the propagated states through the weighted cross correlation of the points. Each $X_{k-1, i}$ point is propagated using the function $f(x(k-1), u(k))$ and the input $u(k)$, where they model the evolution of the states as:

$$
X_{k, i}=f\left(X_{k-1, i}, u(k)\right)
$$

A weighted mean $\hat{x}_{k}$ and covariance $\hat{P}_{x x}$ are obtained from the propagated points, as:

$$
\begin{aligned}
\hat{x}_{k} & =\sum_{i=0}^{2 n} w_{i} \cdot X_{k, i} \\
\hat{P}_{x x} & =\sum_{i=0}^{2 n} w_{i} \cdot\left(X_{k, i}-\hat{x}_{k}\right) \cdot\left(X_{k, i}-\hat{x}_{k}\right)^{T}+Q
\end{aligned}
$$

where the weight $w_{i}$ is [9]:

$$
w_{i}= \begin{cases}\frac{\lambda}{n+\lambda} & i=0, \text { mean } \\ \frac{\lambda}{n+\lambda}+\left(1-\alpha^{2}+\beta\right) & i=0, \text { covariance } \\ \frac{\lambda}{2 \cdot(n+\lambda)} & i>0\end{cases}
$$

where $\beta$ is a parameter that allows to incorporate the a priori knowledge of the distribution of $x$. For a Gaussian distribution $\beta=2$ is optimal. The values of $\hat{x}_{k}$ and $\hat{P}_{x x}$ are the estimations of the evolution of the probability distribution.

\section{Measurement Update}

In the presence of any measurement (ZUPT, ZARU, etc.), we will estimate the effect of the small changes in the states with respect to the observations. The observation mean and 


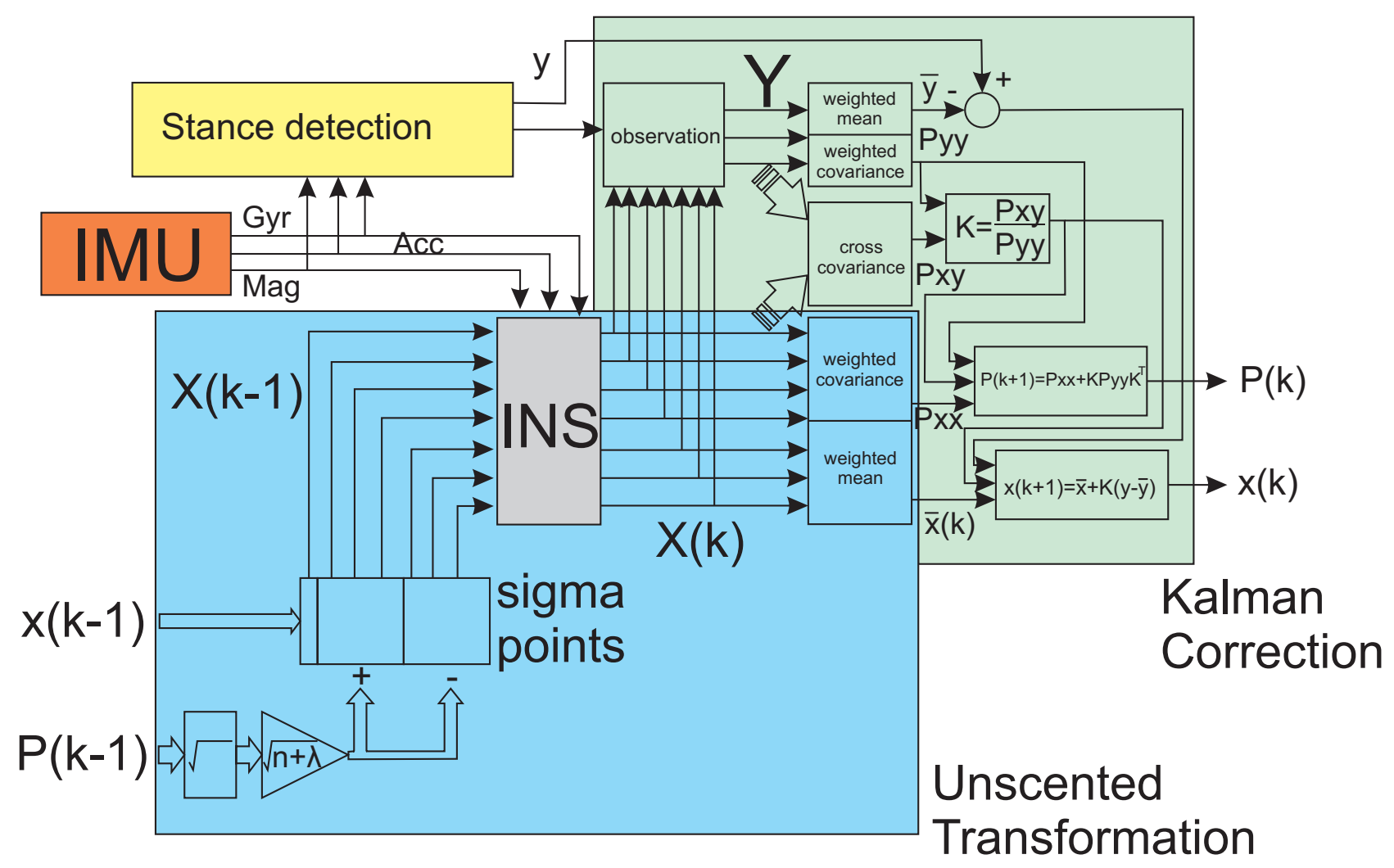

Fig. 1. Structure of a PDR algorithm in an UKF with ZUPT.

covariance are computed evaluating the propagated "sigma" points with the observation function $h()$ as:

$$
Y_{i}=h\left(X_{k, i}\right)
$$

The mean value $\hat{y}$, the covariance of the observation $P_{y y}$ and the covariance between the states and the measurement $P_{x y}$ are calculated as follows:

$$
\begin{aligned}
\hat{y} & =\sum_{i=0}^{2 n} w_{i} \cdot Y_{i} \\
P_{y y} & =\sum_{i=0}^{2 n} w_{i} \cdot\left(Y_{i}-\hat{y}\right) \cdot\left(Y_{i}-\hat{y}\right)^{T}+R \\
P_{x y} & =\sum_{i=0}^{2 n} w_{i} \cdot\left(X_{k, i}-\hat{x}_{k}\right) \cdot\left(Y_{i}-\hat{y}\right)^{T} .
\end{aligned}
$$

Finally, we can update the states with the real measurement $y$ as:

$$
\begin{aligned}
K & =P_{x y} \cdot P_{y y}^{-1} \\
\bar{x}_{k} & =\hat{x}_{k}+K \cdot(y-\hat{y}) \\
P_{k} & =\bar{P}_{x x}-K \cdot P_{y y} \cdot K^{T} .
\end{aligned}
$$

\section{D. $U K F$ in $P D R$}

One of the most common states representation used in an EKF for PDR is a 15 error states representation, where the modeled states are the orientation errors in the navigation frame, the bias in the gyroscope, the error in the position, the error in the velocity and the bias in the accelerometer (each of them in 3 dimensions). This representation is used to generate a more linear problem and diminish the propagation errors. However in the case of the UKF we can use the direct navigation states: Orientation $\Psi$ as Euler angles (Roll $\phi$, Pitch $\theta$ and Yaw $\psi$ ), position $r$ and velocity $\dot{r}$ and the biases of the instrument $(\Delta a$ and $\Delta \omega)$, although any other reduced states can be used. Hence, our state vector will be:

$$
x(k)=\left[\begin{array}{lllll}
\Psi^{T} & \Delta \omega^{T} & r^{T} & \dot{r}^{T} & \Delta a^{T}
\end{array}\right]^{T} .
$$

The structure of the filter can be observed in the Fig. 1. In our implementation we propagate each sigma point using the values of the measured turn rate and acceleration and we can estimate the mean and covariance of the states according to the distribution of the points. Observing the signals from the accelerometer and gyroscope, we can determine the step phases. During the swing, we will use the estimated mean and covariance of the UT. During a stance, we observe the estimated states $X(k)$ according to the desired measurements $\left(Y_{Z U P T, i}=\dot{r}(k, i)\right.$ for ZUPT and $Y_{Z A R U, i}=\Delta \omega(k, i)$ for ZARU) and determine the estimated mean, the covariance of the measurements and the correlation between the changes in the states and the changes in the measurements. Using equations 16 and 17 we can obtain the corrected states.

For the evaluation of the UKF we used the data set 14 of [17]. The signal was recorded from an IMU placed in the 

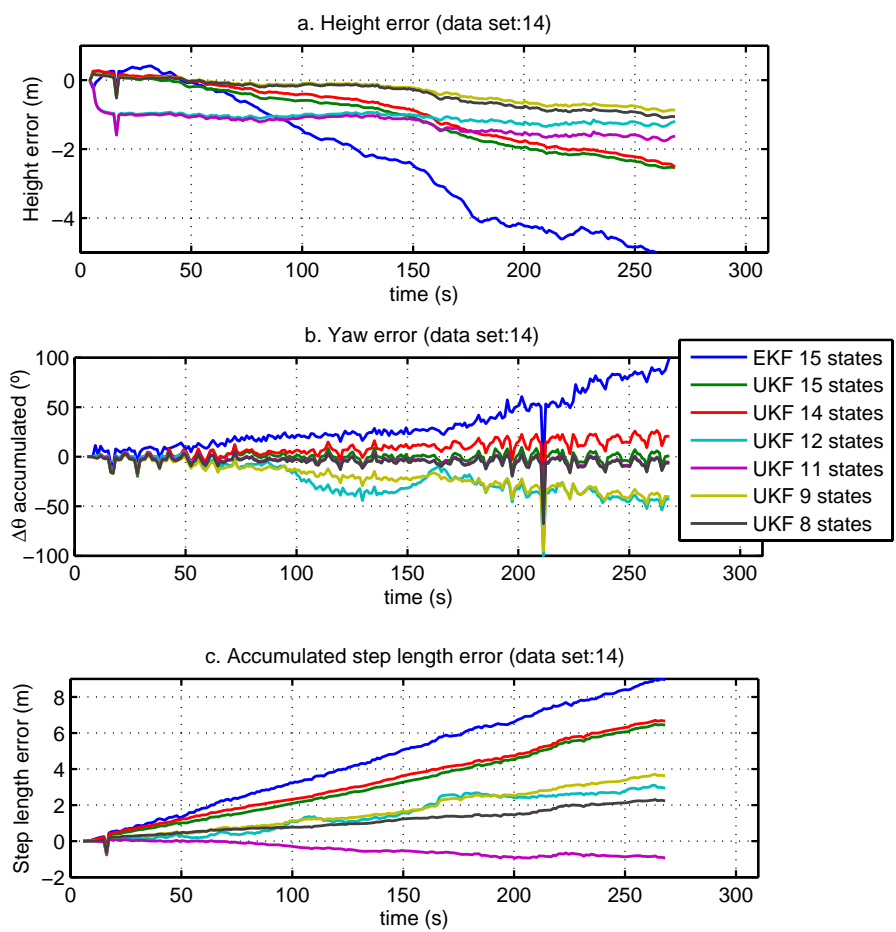

Fig. 2. Analysis of the height (a), orientation (b) and step length (c) errors for the data set 14 of [17].

instep of the right foot, while doing a 5 minutes closed loop trajectory with 3 seconds of stance before the first movement. The ground truth was recorded with a camera motion system. In this work we propose an evaluation criterion based on the height error, the yaw error (the accumulation of the heading change error $\Delta \theta$ ) and the step length accumulated error. We evaluated the following representations:

- 15 states: full state representation, with ZUPT and ZARU.

- 14 states: state representation without the yaw state, with ZUPT and ZARU.

- 12 states: state representation without the accelerometer bias states, with ZUPT and ZARU.

- 11 states: state representation without the accelerometer bias states nor the yaw state, with ZUPT and ZARU.

- 9 states: state representation with none of the bias states, with ZUPT only.

- 8 states: state representation with none of the bias states nor the yaw state, with ZUPT only.

In the figure 2, we can observe that the UKF behaves better than the EKF in most conditions, particulary in 15 and 9 states versions. From now and on, we will use the 15 states UKF filter when referring to the UKF.

\section{Magnetic Angular Rate Update (MARU)}

Many IMUs usually have a magnetometer and accordingly, many PDR implementations such as in [3], [11] and [18]

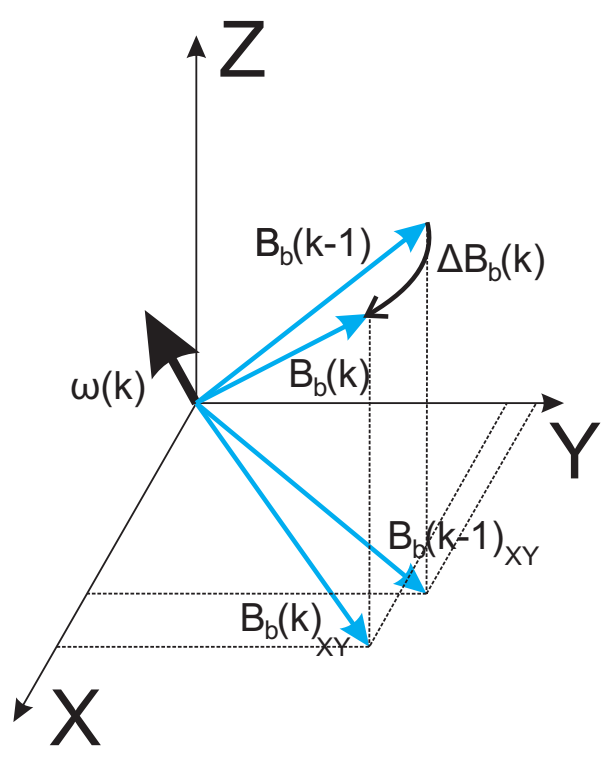

Fig. 3. Change in the measured magnetic field vector due to a rotation, represented in the sensor's reference frame.

make use of that measurement to provide information about the orientation. Indoors, the presence of magnetic disturbances usually causes local errors in the yaw that due to the integration can generate high positioning errors. Today's buildings are usually constructed using metallic structures, and that generates strong position dependant magnetic perturbations.

We propose to use the change in the magnetic filed to provide a measurement of the turn rates of the sensor. This will allow us to use any magnetic field that is constant with time, which can be assumed during the stance phase in a foot-mounted IMU. If we rotate an IMU while the magnetic field is constant in the navigation frame, we will observe a rotation of the measured magnetic field as it can be seen in the Fig. 3. In the figure $B_{b}(k)$ and $B_{b}(k-1)$ represent the actual and previous measured magnetic field, $\omega(k)$ the turn rate of the sensor and $\Delta B_{b}(k)$ the resulting change in the measured magnetic field. We will call this measurement Magnetic Angular Rate Update (MARU).

We plan to use this measurement as information to limit the drift in the yaw, provide a better attitude propagation and a better Gyroscope bias estimation. Due to the nonlinearities present in the attitude propagation and measurement relationships, we can use the UKF, but in the case of a EKF, a first order approximation is needed.

In this work we establish three strategies to use the magnetic field changes as information for the turn rates:

A. Change in the magnetic heading: This approach associates the change in the magnetic heading (the magnetic field in the horizontal plane) with the change in the estimated yaw and therefore with the turn rate in the vertical axis.

B. Magnetic rotation: Using an UKF, it is possible to directly associate the turn rates and the magnetic field 
changes in a three dimensional space and therefore obtain information on the attitude change and the turn rates.

C. Magnetic rotation first order approximation: A linear approximation can be used in an EKF, to implement the relationship of the magnetic rotation with the Gyroscope biases as a measurement.

The use of any of these methods will depend on the desired processing effort and the states implemented. The presence of changing magnetic fields (moving metal objects, motors, etc.) might alter the measurements and therefore, it is necessary to establish a threshold as a way to minimize this effect. This threshold will be discussed for each of the three cases next.

\section{A. Change in the magnetic heading}

A first approach to take advantage of the magnetic field changes is the use of the magnetic heading. The use of ZUPT measurements provide information about the roll an pitch but the yaw $\psi$ remains unobserved. During a stance the changes of the magnetically detected yaw must be correlated with the changes in the INS orientation, therefore we will use that information to decrease the error in the yaw.

We propose the use of the change in the angle in the horizontal plane from the magnetic field to the $\mathrm{X}$ axis of the sensor frame (IMU heading) during the stance as a measurement of the change in the yaw as observed in the Figure 4. Transforming the magnetic field measurements from the sensor frame to a horizontal plane (correcting the roll $\phi$ and pitch $\theta$, subindex $n^{\prime}$ ) as:

$$
B_{n^{\prime}}(k)=R_{\theta} \cdot R_{\phi} \cdot B_{b}(k),
$$

where:

$$
\begin{aligned}
& R_{\theta}=\left[\begin{array}{ccc}
\cos \theta(k) & 0 & \sin \theta(k) \\
0 & 1 & 0 \\
-\sin \theta(k) & 0 & \cos \theta(k)
\end{array}\right], \\
& R_{\phi}=\left[\begin{array}{ccc}
1 & 0 & 0 \\
0 & \cos \phi(k) & -\sin \phi(k) \\
0 & \sin \phi(k) & \cos \phi(k)
\end{array}\right] .
\end{aligned}
$$

we can obtain the magnetic yaw as:

$$
\psi_{m}(k)=-\arctan \left(B_{n^{\prime}}(k)_{y}, B_{n^{\prime}}(k)_{x}\right)-M_{d} .
$$

The observation function $Y()$ and the measurement value $y()$ for the UKF will be:

$$
\begin{aligned}
\Delta \psi_{m}(k)= & \Psi_{m}(k)-\Psi_{m}(k-1) \\
y(k)= & \arctan \left(B_{n^{\prime}}(k-1)_{y}, B_{n^{\prime}}(k-1)_{x}\right) \\
& -\arctan \left(B_{n^{\prime}}(k)_{y}, B_{n^{\prime}}(k)_{x}\right) \\
Y(k)= & \psi(k)-\psi(k-1) .
\end{aligned}
$$

For the estimation of the observation on each point we must use the previous and the propagated yaw value, $\psi(k-1)$ and $\psi(k)$ respectively, corresponding to the evaluated "sigma" point. The difference between them will be related to the change in the magnetic heading. This is implemented using an UKF. The general structure of the implemented UKF can be observed in Figure 1.

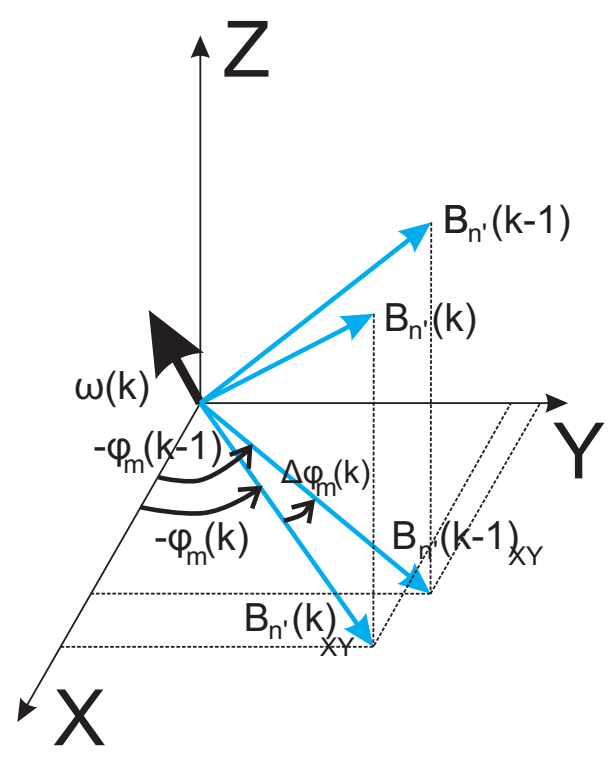

Fig. 4. Change in the magnetic heading due to the turn rates observed in $B_{n^{\prime}}$ in the modified navigation frame (estimated horizontal plane).

For the detection of the MARU with the change in the magnetic heading we must assume that the IMU is in a stance phase, where we assumed a limit $\gamma_{a}$ for the norm of the measured acceleration $(A c c)$ and a limit $\gamma_{\omega}$ for the norm of the measured turn rate $(G y r)$. The change in the yaw must not be over the maximal change permitted with that value, therefore the limit $\gamma_{\psi}$ for the norm of the change of the heading must be:

$$
\gamma_{\psi}=\gamma_{\omega} \cdot \Delta t
$$

Adding this to the stance assumption, the condition $T_{m h}$ to use the MARU measurement with the change in the magnetic heading becomes:

$$
\begin{aligned}
T_{m h}\left(\text { Acc }, \text { Gyr }, \Delta \psi_{m}\right)= & \left(\|A c c\|<\gamma_{a}\right) \&\left(\|G y r\|<\gamma_{\omega}\right) \\
& \&\left(\left\|\Delta \psi_{m}(k)\right\|<\gamma_{\psi}\right) .
\end{aligned}
$$

\section{B. Magnetic rotation}

Observing the rotation of the magnetic field due to an turn rate can provide more information about the turn rates, therefor we will study the change of the measured magnetic field in the sensor frame

The change in the measured magnetic field $\Delta B_{b}(k)$ is:

$$
\Delta B_{b}(k)=B_{b}(k)-B_{b}(k-1) .
$$

Transforming the measurements from the sensor frame to the navigation frame using $B_{b}(k)=C_{b}^{n}(k)^{T} \cdot B_{n}(k)$, we obtain:

$$
\Delta B_{b}(k)=C_{b}^{n}(k)^{T} \cdot B_{n}(k)-C_{b}^{n}(k-1)^{T} \cdot B_{n}(k-1) .
$$

The Direction Cosine Matrix $C_{b}^{n}(k)$ can be rewritten as its propagation from the previous value (eq. 3 ), therefore the 
relationship will be:

$$
\begin{aligned}
\Delta B_{b}(k)= & \left(C_{b}^{n}(k-1) \cdot e^{\Omega_{n b}^{b}(k) \cdot \Delta t}\right)^{T} \cdot B_{n}(k) \\
& -C_{b}^{n}(k-1)^{T} \cdot B_{n}(k-1) \\
= & e^{-\Omega_{n b}^{b}(k) \cdot \Delta t} \cdot C_{b}^{n}(k-1)^{T} \cdot B_{n}(k) \\
& -C_{b}^{n}(k-1)^{T} \cdot B_{n}(k-1) .
\end{aligned}
$$

The magnetic field in the navigation frame during the moment $k$ can be written as $B_{n}(k)=B_{n}(k-1)+\varepsilon_{B}$, where $\varepsilon_{B}$ is the change in the magnetic disturbance. Therefore, equation 30 can be rewritten as:

$$
\begin{aligned}
\Delta B_{b}(k)= & e^{-\Omega_{n b}^{b}(k) \cdot \Delta t} \cdot C_{b}^{n}(k-1)^{T} \cdot\left(B_{n}(k-1)+\varepsilon_{B}\right) \\
& -C_{b}^{n}(k-1)^{T} \cdot B_{n}(k-1) \\
= & \left(e^{-\Omega_{n b}^{b}(k) \cdot \Delta t}-I\right) \cdot C_{b}^{n}(k-1)^{T} \cdot B_{n}(k-1) \\
& +e^{-\Omega_{n b}^{b}(k) \cdot \Delta t} \cdot C_{b}^{n}(k-1)^{T} \cdot \varepsilon_{B} .
\end{aligned}
$$

Using the fact that $C_{b}^{n}(k-1)^{T} \cdot B_{n}(k-1)=B_{b}(k-1)$ and $e^{-\Omega_{n b}^{b}(k) \cdot \Delta t} \cdot C_{b}^{n}(k-1)^{T}=\left(C_{b}^{n}(k-1) \cdot e^{\Omega_{n b}^{b}(k) \cdot \Delta t}\right)^{T}=$ $C_{b}^{n}(k)^{T}$, the change in the sensor magnetic field will be:

$$
\Delta B_{b}(k)=\left(e^{-\Omega_{n b}^{b}(k) \cdot \Delta t}-I\right) \cdot B_{b}(k-1)+C_{b}^{n}(k)^{T} \cdot \varepsilon_{B} .
$$

This equation relates the change in the measured magnetic field $\Delta B_{b}(k)$ with the turn rates $\omega(k)$ (implicit in $\Omega_{n b}^{b}(k)$ ).

If the foot is on a stance phase, any position dependant perturbation will be constant and we can assume that $\varepsilon_{B}$ will be caused only by the random noise of the Magnetometer. We propose the use of the UKF represented in Figure 1 and the expression from equation 5 for $e^{-\Omega_{n b}^{b}(k) \cdot \Delta t}$, discarding $\varepsilon_{B}$ to propagate the attitude and estimate the Gyroscope bias. Combining equation 5 and equation 32 results in:

$$
\begin{aligned}
\Delta B_{b}(k)= & \left(\frac{(1-\cos (\|\omega(k) \Delta T\|))}{\|\omega(k)\|^{2}} \Omega_{n b}^{b}(k)^{2}\right. \\
& \left.-\frac{\sin (\|\omega(k) \Delta T\|)}{\|\omega(k)\|} \Omega_{n b}^{b}(k)\right) \cdot B_{b}(k-1)
\end{aligned}
$$

where $\omega(k)$ represents the turn rate after eliminating the bias, $\omega(k)=\operatorname{Gyr}(k)-\Delta \omega(k)$. The measurement $y(k)$ and the observation function $Y(k)=h(k)$ are:

$$
\begin{aligned}
y(k)= & B_{b}(k)-B_{b}(k-1) \\
Y(k)= & \left(\frac{(1-\cos (\|\omega(k) \Delta T\|))}{\|\omega(k)\|^{2}} \Omega_{n b}^{b}(k)^{2}\right. \\
& \left.-\frac{\sin (\|\omega(k) \Delta T\|)}{\|\omega(k)\|} \Omega_{n b}^{b}(k)\right) \cdot B_{b}(k-1) .
\end{aligned}
$$

As a way to avoid the changes in the magnetic disturbances we propose to limit the norm of the change in the magnetic field $\left\|\Delta B_{b}\right\|$ to the arc formed by the vector $B_{b}$ when the turn rate is perpendicular to it. Using the threshold $\gamma_{\omega}$ for the turn rate during the time interval $\Delta t$, the limit $\gamma_{B}$ for the change in the magnetic field will be:

$$
\gamma_{B}=\gamma_{\omega} \cdot \Delta t \cdot B_{b}(k-1)
$$

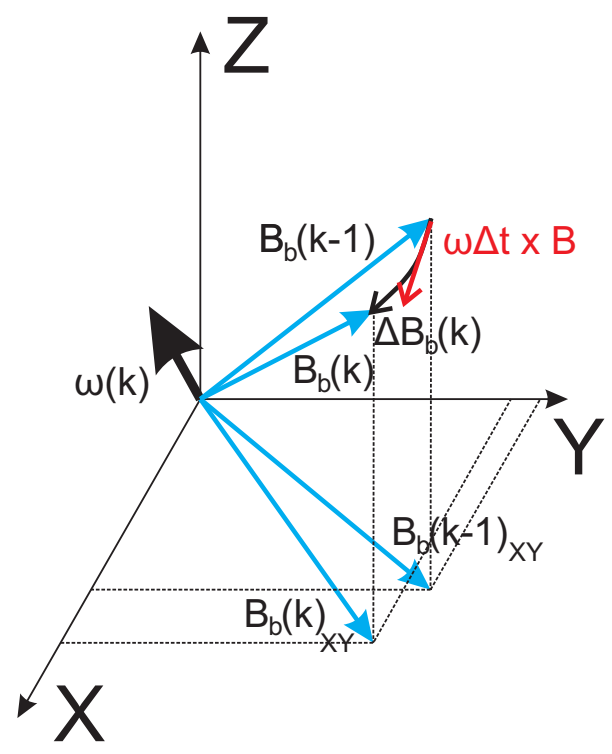

Fig. 5. Change in the measured magnetic field vector $B_{b}$ due to a rotation, represented in the sensor's reference frame. In red the first order approximation of the increment.

Adding this to the stance assumption, the condition $T_{m r}$ to use the MARU measurement with magnetic rotations will be:

$$
\begin{aligned}
T_{m r}\left(\text { Acc, Gyr, } \Delta B_{b}(k)\right)_{k}= & \left(\|A c c\|<\gamma_{a}\right) \&\left(\|G y r\|<\gamma_{\omega}\right) \\
& \&\left(\left\|\Delta B_{b}(k)\right\|<\gamma_{B}\right) .
\end{aligned}
$$

\section{Magnetic rotation first order approximation}

Although the UKF offers a better behavior for non-linear systems it might be necessary to implement an EKF. In this case we are going to propose a linearized form of equation 32 . The first order approximation of $e^{-\Omega_{n b}^{b}(k) \cdot \Delta t}$ is:

$$
\begin{aligned}
e^{-\Omega_{n b}^{b}(k) \cdot \Delta t} & =I-\Omega_{n b}^{b}(k) \cdot \Delta t \\
& =I-[\omega(k) \Delta t \quad \times] .
\end{aligned}
$$

This correspond to the red vector in the figure 5 , a linear approximation of the movement of the vector $B_{b}$ according to the turn rate. Inserting this approximation in equation 32 we obtain:

$$
\Delta B_{b}(k)=-[\omega(k) \Delta t \quad \times] \cdot B_{b}(k-1)+C_{b}^{n}(k)^{T} \cdot \varepsilon_{B} .
$$

Adding the effect of the bias in the Gyroscope $(\omega(k)=$ $\operatorname{Gyr}(k)-\Delta \omega(k))$ to the previous equation results in:

$$
\begin{aligned}
\Delta B_{b}(k)= & -[(G y r(k)-\Delta \omega(k)) \Delta t \quad \times] \cdot B_{b}(k-1) \\
& +C_{b}^{n}(k)^{T} \cdot \varepsilon_{B} \\
= & -\left[\begin{array}{ll}
G y r(k) \Delta t & \times
\end{array}\right] \cdot B_{b}(k-1)+C_{b}^{n}(k)^{T} \cdot \varepsilon_{B} . \\
& +\left[\begin{array}{ll}
\Delta \omega(k) \Delta t & \times
\end{array}\right] \cdot B_{b}(k-1)
\end{aligned}
$$

Reordering the equation to represent a linear relationship between the measurement and $\Delta \omega(k)$ leads to:

$$
\begin{aligned}
& \Delta B_{b}(k)+[\operatorname{Gyr}(k) \Delta t \quad \times] \cdot B_{b}(k-1)= \\
& {\left[\begin{array}{ll}
-B_{b}(k-1) \Delta t & \times
\end{array}\right] \cdot \Delta \omega(k)+C_{b}^{n}(k)^{T} \cdot \varepsilon_{B} .}
\end{aligned}
$$


The measurement $m(k)$ and the observation matrix $H(k)$ (for a 15 states EKF) becomes;

$$
\begin{aligned}
& m(k)=\Delta B_{b}(k)+[\operatorname{Gyr}(k) \Delta t \quad \times] \cdot B_{b}(k-1) \\
& \left.H(k)=\left[\begin{array}{llll}
0_{3 x 3} & {\left[-B_{b}(k-1) \Delta t\right.} & \times
\end{array}\right] \quad 0_{3 x 9}\right] .
\end{aligned}
$$

It is possible to detect the measurement using the same threshold established in equation 36 and 37. This observation matrix is the same as the one obtained in [12] and [19] but the generality of the method, the detection and the implementation differ.

\section{MARU evaluation}

For the evaluation of the MARU method we used the synthetic noiseless IMU signals proposed in [16] adding magnetic dipoles patterns to account for the magnetic perturbations of a normal building. We observed that magnetically aided PDR methods (using the magnetometer as a compass), as the one proposed in [11], tend to accumulate errors in the position after each turn. However, using the MARU measurements provided a more accurate reconstruction of the position.

Using the noiseless signals we propose to do a Monte Carlo evaluation of the MARU measurement, adding noise patterns to the accelerometer, gyroscope and magnetometer to generate 100 different simulations. We will base our evaluation on 2 error figures, the root mean squared position error $e_{r}(t)$ :

$$
e_{r}(t)=\sqrt{\frac{1}{N} \cdot \sum_{i=1}^{N} \sum_{n=x, y, z}\left(r_{n, i}(t)-r_{n}(t)\right)^{2}}
$$

and the root mean squared yaw error $e_{\psi}(t)$ :

$$
e_{\psi}(t)=\sqrt{\frac{1}{N} \cdot \sum_{i=1}^{N}\left(\psi_{i}(t)-\psi(t)\right)^{2}} .
$$

The evolution of the root mean squared errors can be observed in figure 6 for different filter approaches, where we can see that the MARU is able to obtain information about the gyroscope bias during the stance phases and therefore is able to lower the error trends. In the first seconds of the experiment we can observe a higher error of the yaw. This is due to the fact that we are adding noise from the new measurements, but after 100 seconds the error decreases. The method that reduces the error the most is the magnetic rotation for the UKF (UKF MARU2, magenta line in figure 6) due to the fact that it obtains more information about the biases during the stances. We also have observed that if a ZARU establishes a value for the biases in the gyroscope, then the MARU effect is minimal and it is observed only after several minutes.

\section{Soft Measurements}

We have observed that during a normal walk, the IMU presents slow movements phases before and after the stance. This slow movement detections can be confused with the zero velocity phase, and must be handled. Usually, the step detection algorithms discard this sub phases to avoid adding errors in the ZUPT correction. However, this reduces the
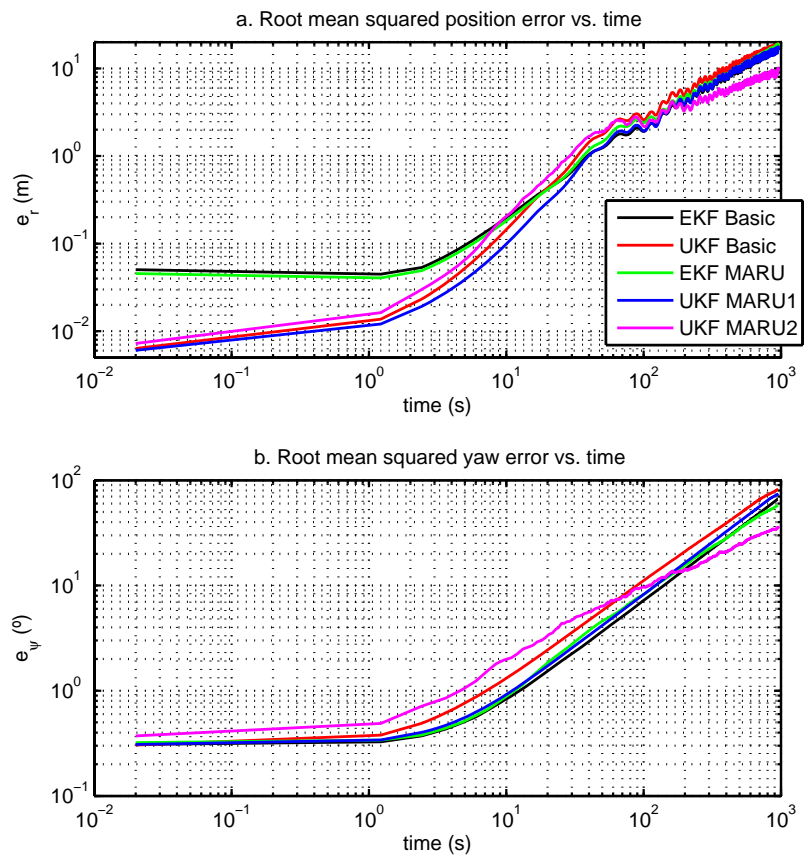

Fig. 6. Evolution of the root mean squared errors in the position (a) and yaw (b) versus time. MARU1 refers to the change in the magnetic heading and MARU2 refers to the magnetic rotation. EKF MARU uses the linear approximation of the magnetic rotation.

amount of information that can be used for corrections and in some cases like running, the stances might not be detected. It would be beneficial to make the detection of the change between the swing and the stance phases not abrupt since it will allow the use of more measurements. Therefore we propose to establish a wider stance window with transitions at the beginning and the end to account for the pre and post stance phases.

\section{A. Veracity of the stance}

In the Fig. 7 we can observe 2 step detection algorithms with respect to the IMU signals (the gyroscope and stance detection signals was amplified for an easier view): the first (magenta line in Fig. 7) is the retarded step detection proposed in [16] and the second (black line in Fig. 7) uses only thresholds for the magnitude of the acceleration and turn rates. The last one provides more samples as information, but the information might not be as good as in the first case. It is clear that the veracity of the zero velocity assumption is zero before the pre stance, and it starts to grow until it reaches its maximal at the center of the stance phase. Then it starts to decrease until the end of the post stance phase.

We propose the generation of a veracity signal measuring the amount of step detections passed in an evaluation window with the size of the stance zone; a higher the amount of detections in the vicinity of the sample will imply a higher veracity of the detection. This will require the signal to be post processed, but we can still obtain good results using 

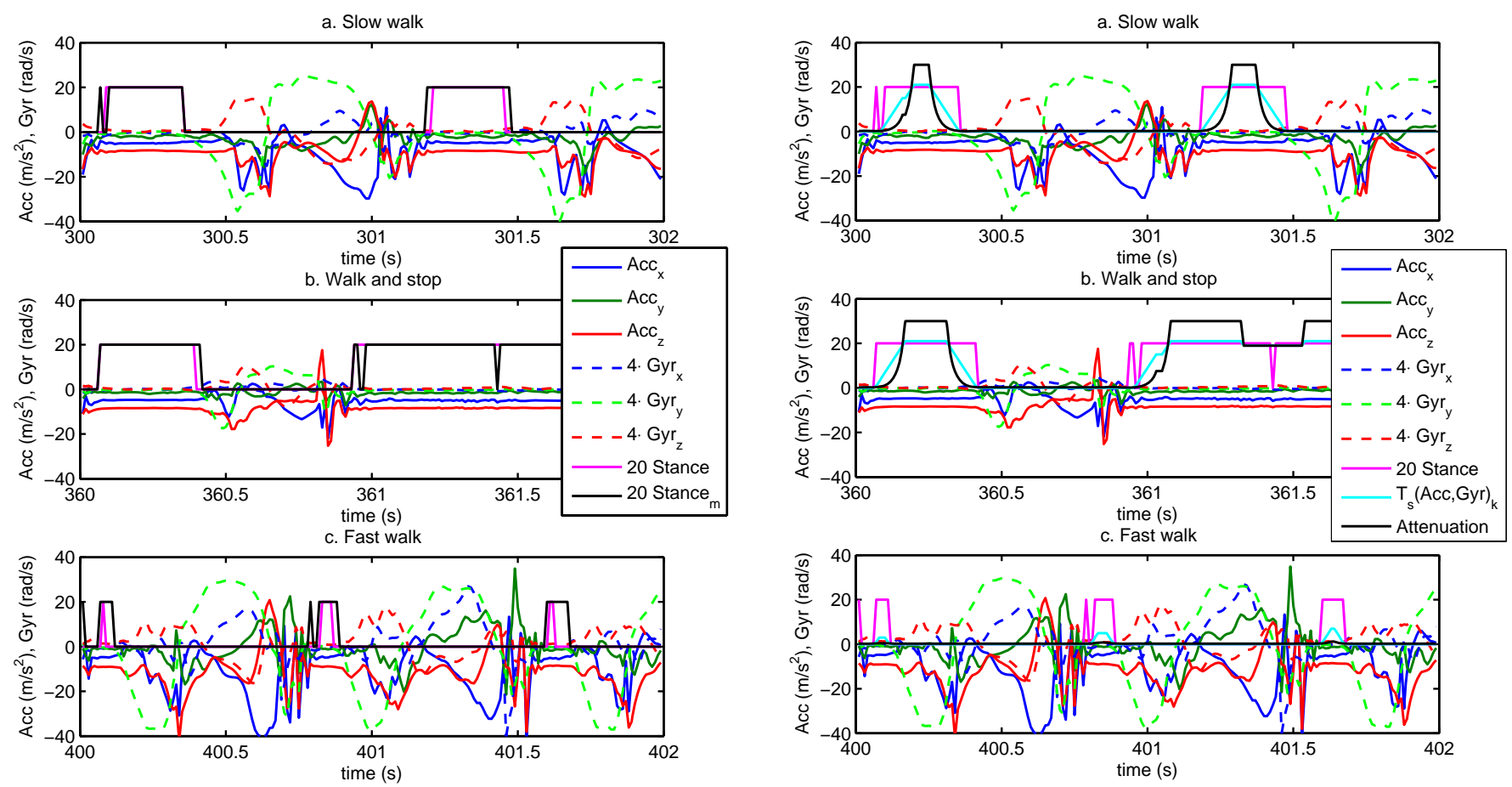

Fig. 7. Acceleration, turn rate (amplified) and results of 2 stance detection methods used during normal walk (a), walk and detention (b) and fast walk/running (c), data obtained from data set 15 of [17].

a delay of $N$ samples and therefore an evaluation window of $2 N+1$ samples. We can measure the number of stance detections in the window $l(k)$ using $\left(T(A c c, G y r)_{k}=1\right.$ in stance and $T(A c c, G y r)_{k}=0$ in swing):

$$
l(k)=\sum_{j=-N}^{N} T(\text { Acc }, \text { Gyr })_{k+j} .
$$

Our new window will have $N^{\prime}$ samples so $2 N^{\prime}+1=l(k)$. A rising (beginning of the stance) and falling (last samples of the stance) slope can be obtained with:

$T_{s}(\text { Acc }, \text { Gyr })_{k}=\max \left(\sum_{j=-N^{\prime}}^{N^{\prime}}\left(2 \cdot T(A c c, G y r)_{k+j}-1\right), 0\right)$,

In the cases of more swing detections than stance detections $T_{s}(A c c, G y r)_{k}$ will be 0 . An example of a step detection threshold that is used to generate the signal "Stance $m$ " in the Fig. 7 is:

$T(\text { Acc, Gyr })_{k}=\left(\left\|\left(\left\|A c c_{k}\right\|-9.8\right)\right\|<\gamma_{a}\right) \&\left(\left\|G y r_{k}\right\|<\gamma_{\omega}\right)$.

The veracity signal can be observed in the light blue line of the figure 8 . This signal is able to provide step detections even when the pedestrian is running, but indicates that the most important information for zero velocity updates or ZARU, MARU, etc. is available in the center of the stance. The points

Fig. 8. Acceleration, turn rate (amplified), stance detection, veracity and attenuation to the measure covariance during normal walk (a), walk and detention (b) and fast walk/running (c), data obtained from data set 15 of [17].

with a high veracity are points in which it is certain that the stance condition is true and therefore in the case of the ZUPT the velocity is closer to zero and in the case of the MARU the the magnetic field in the navigation frame is more constant. On the opposite case if the veracity is low the stance condition is not as certain and the previous conditions are not that strong.

The covariance of the estimated zero velocity or zero change of the magnetic field in the navigation frame, are referred in the covariance of the measurement ( $R$ in equation 13), therefore the value of $R$ must be related with the veracity. To associate the veracity with the covariance of the measurement, we tried several functions (lineal, inverse, exponential, etc.) and the best results for the resulting covariance of the measurement $R(k)$ were obtained with:

$$
R(k)=10^{\frac{T_{s, \max }-T_{s}(A c c, G y r)_{k}}{m_{s} \cdot T_{s, \max }}} \cdot R_{0}
$$

where $T_{s, \max }$ is the maximal value of $T_{s}(\text { Acc, Gyr })_{k}, m_{s}$ represents an attenuation factor for low veracities and $R_{0}$ is the covariance of the measurement when the veracity is maximum. In figure 8 we can observe in black a proposed exponential attenuation function to apply to the covariance of a measurement. The same conditions of figure 7 were used to generate figure 8. 


\section{B. Evaluation of the soft measurements}

For the evaluation of the soft measurements we used the data set 16 of [17]. The data was recorded from an IMU placed in the instep of the right foot, while doing a 5 minutes closed loop running/walking trajectory without a long stance before the first movement. The ground truth was recorded with a camera motion system and we tested the EKF and the UKF (with their respective versions of MARU) with and without the soft measurements.

From figure 9, we can see that the soft measurements applied in the covariances of the ZUPT and MARU, decreased the errors in most cases. This is because it uses more measurement samples per step and therefore it obtains more information. In this example the best results are obtained using the UKF with MARU1 (change in the magnetic heading) and soft measurements.

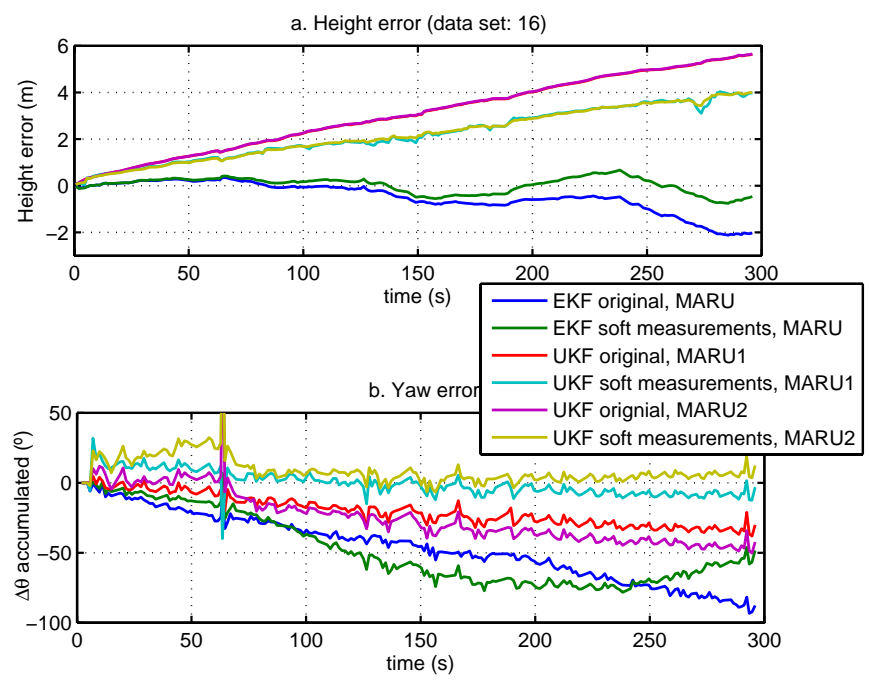

c. Accumulated step length error

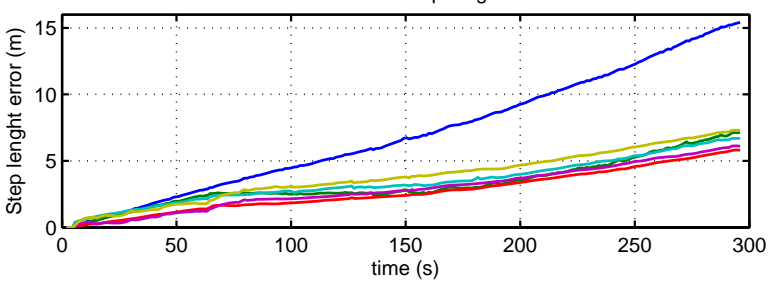

Fig. 9. Analysis of the height (a), orientation (b) and step length (c) errors for the data set 16 of [17] with several algorithms, with and without soft decisions. MARU1 refers to the change in the magnetic heading and MARU2 refers to the magnetic rotation. EKF MARU uses the linear approximation of the magnetic rotation

\section{Performance Analysis}

For the evaluation of the introduced approaches we propose 2 trajectories, both are recorder using the MTx IMU from XSens mounted in the instep of the right foot. The first experiment is recorded walking in the first floor of an office building. The floor is covered with carpet and the user is standing 9 seconds before starting the movement, in order to generate a
Trajectory around the first floor of the TE01 building in DLR

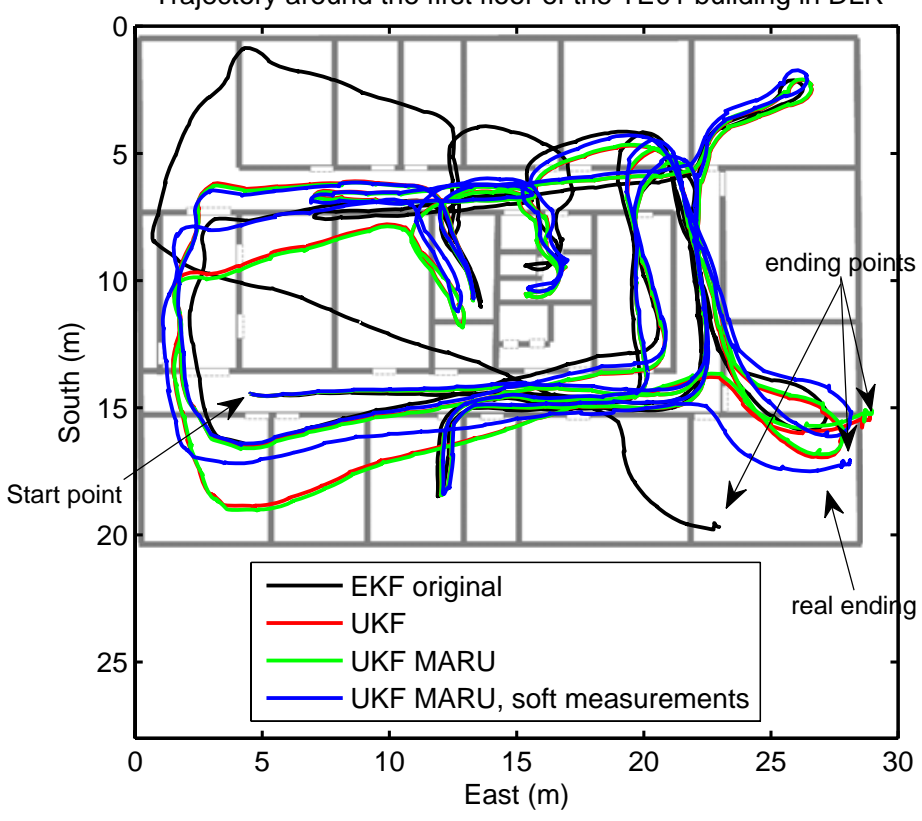

Fig. 10. Several estimated trajectories for a PDR reconstruction of a walk in the first floor of an office building. MARU uses the magnetic rotation approach.

ZARU measurement and estimate the gyroscope biases. The trajectory starts in a rectangular corridor, walking East, then it follows the corridor of the building counterclockwise, entering in some of the offices and ending in the South East office. The experiment lasted 5 minutes.

In figure 10, we can see the estimation of the trajectories obtained after using several PDR methods. We can observe that the UKF reduces the errors in the estimation of the orientation compared to the EKF. Adding the MARU does not affect significantly the estimation because of the initial ZARU. The UKF with MARU (using magnetic rotation) and soft measurements provides the best estimate of the position. We assume that it is due to the soft measurements of the ZUPT which improves the behavior of the zero velocity update in the carpet and provides more information to the MARU.

The second experiment consists of a closed loop trajectory in a different office building. The user is standing 10 seconds before starting the movement in order to generate a ZARU measurement and estimate the gyroscope biases. The trajectory starts entering the building and follows a counterclockwise path passing through some of the offices. After the first round in the building, the subject exits the building and follows a rectangular path in the parking lot. Finally the first round is repeated and the user ends in the same starting point. The trajectory lasted 10 minutes.

In figure 11, we can see the estimation of the trajectories after using several PDR methods. We can observe that the UKF reduces the errors associated with the loss of the heading. The introduction of the MARU measurements generate an initial 


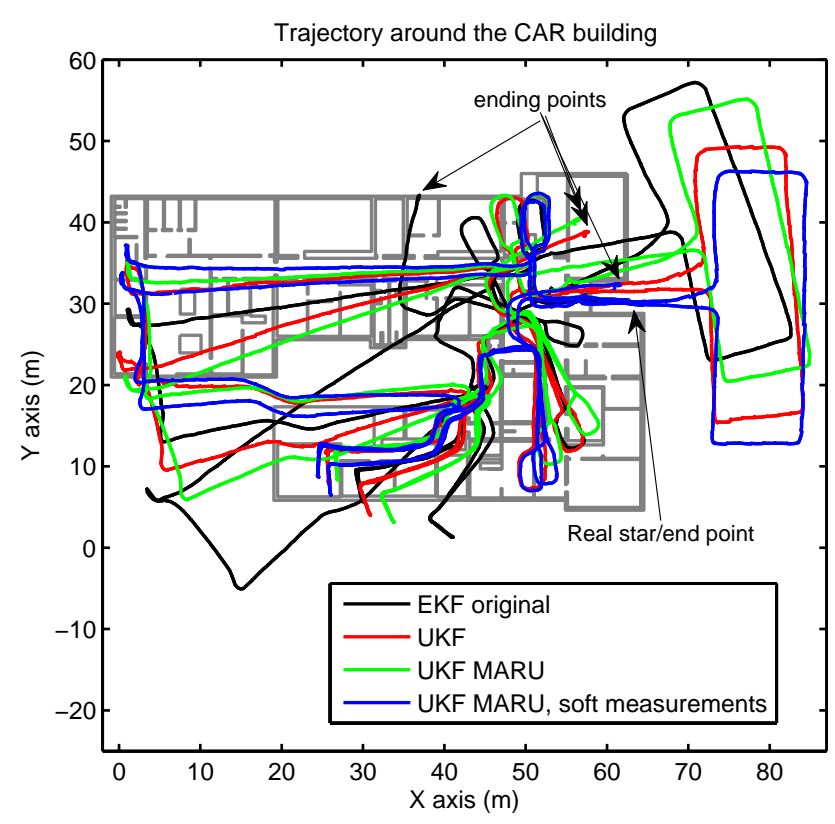

Fig. 11. Several estimated trajectories for a PDR reconstruction of a walk in the A building of the Centre for Automation and Robotics. MARU refers to the change in the magnetic heading.

loss of the heading, mainly due to the introduction of the magnetometer errors. However, after some time the orientation is maintained in a better way, although the initial errors is still present. If the trajectory lasted longer we would see a constant error in this case. By adding the soft measurements, we are able to estimate the errors in the system faster, and correctly propagate the orientation.

We can observe that the UKF provides better results than the EKF, however with the UKF we observed higher estimation times. As a way to measure the computational load required for using the proposed methods, we compared the algorithms execution times using MATLAB implementations in a $3.2 \mathrm{GHz}$ Pentium 4, 4 GB RAM PC using Windows XP. We avoided running other programs during the test. We used the following 3 datasets with $100 \mathrm{~Hz}$ sampling rate:

- Data set 14 of [17]: a 280.61 seconds walking scenario.

- Data set 17 , recorded using the technique proposed in [17]: a 657.04 seconds walking/running scenario.

- Synthetic signal: a 981.60 seconds simulation of walking in a closed path.

In table I, we list the computation times and the percentage of the signal time required for the methods implemented in this work. It is clear that even if the UKF provides a more accurate estimations, it needs more computation time than other methods. This is mainly due to the multiple propagations needed to measure the evolution of the "sigma" points. This disadvantage however might be compensated by the advances in parallel computing that have taken place. The usage of MARU or soft measurements only represent around $10 \%$ additional computing load.

\begin{tabular}{|c|c|c|c|c|c|c|}
\hline \multirow{2}{*}{$\begin{array}{c}\text { Used } \\
\text { filter }\end{array}$} & \multicolumn{2}{|c|}{ Data set 14} & \multicolumn{2}{c|}{ Data set 17} & \multicolumn{2}{c|}{ Synthetic } \\
\cline { 2 - 7 } & time & $\%$ & time & $\%$ & time & $\%$ \\
\hline EKF & 13.49 & 4.81 & 32.12 & 4.88 & 47.55 & 4.84 \\
\hline EKF MARU & 14.07 & 5.01 & 35.35 & 5.38 & 50.06 & 5.10 \\
\hline EKF MARU s & 16.48 & 5.87 & 38.36 & 5.84 & 59.16 & 6.03 \\
\hline UKF & 121.45 & 43.29 & 304.54 & 46.35 & 445.83 & 45.41 \\
\hline UKF MARU & 144.31 & 51.43 & 337.13 & 51.31 & 486.94 & 49.61 \\
\hline UKF MARU s & 141.13 & 50.29 & 321.57 & 48.94 & 474.08 & 48.30 \\
\hline
\end{tabular}

TABLE I

COMPUTING TIME (IN SECONDS) AND THEIR RESPECTIVE \% OF THE SIGNAL TIME FOR DIFFERENT ESTIMATION METHODS FOR 3 TEST SIGNALS. MARU REFERS TO THE MAGNETIC ROTATION AND "S" TO THE USE OF SOFT MEASUREMENTS IN ZUPT AND MARU.

\section{CONCLUSIONS}

We have presented a new implementation of Pedestrian Dead-Reckoning using the Unscented Kalman Filter instead of the widely used Extended Kalman Filter. The new filter adapts better to the non-linearities of the orientation propagation which allows for better estimations of the position and orientation at the cost of increasing the computation time. The UKF filter also allows the usage of non-linear measurements without the need of linear approximations as in the EKF.

We also presented three turn rate biases estimation methods based on the measured changes in the magnetic field during the stance. These methods improve the estimation, especially when there is no initial long stance phase. Those methods can be used indoors even in the presence of strong position dependant perturbations due to the fact that they use the points where the magnetic perturbations are constant.

Finally, we presented a method to treat the changes between the detections of the stance and the swing, gradually allowing the covariance of the measurements to be adapted according to the veracity of the stance detection. This method also allows the detection of different walking speeds and adapts the covariance of the measurement accordingly.

The previous methods improves the basic PDR algorithm and allow high level fusion algorithms to work better.

Future works may include the usage of quaternion angles in the angle propagation to avoid gimbal lock and other representation problems. It is also possible to change the states to be modeled to generate a orientation estimator based on the UKF. It is important to study a better perturbation change detector for the MARU measurements, because is the main source of error in that measurement. If the perturbations are detected they can be used as references for the position. The veracity-covariance function proposed in this work was obtained based on the results but a statistical analysis of the velocity and magnetic change can be used to obtain a better relationship. It is clear that a Dead-Reckoning will always have an increasing error and therefore global measurements or map matching must be used to limit those errors.

\section{ACKNOWLEDGMENT}

This work was supported by the LEMUR project (TIN2009-14114-C04-03), LAZARO project (CSIC-PIE Ref.201150E039) and DLR internal project Galileo ADvanced 
APplications (GalileoADAP). We would like to thank the CSIC for financing the research exchange stay that promoted this work.

\section{REFERENCES}

[1] D. Titterton and J. Weston, Strapdown Inertial Navigation Technology. 2004.

[2] J. A. Farrell and M. Barth, The global positioning system and inertial navigation. McGraw-Hill, 1999.

[3] Q. Ladetto, V. Gabaglio, B. Merminod, and L. Ag, "Two Different Approaches for Augmented GPS Pedestrian Navigation," in International Symposium on Location Based Services for Cellular Users, Locellus 2001, pp. 1-7, 2001.

[4] E. Foxlin, "Pedestrian tracking with shoe-mounted inertial sensors," IEEE Computer Graphics and Applications, no. December, pp. 38-46, 2005.

[5] S. Rajagopal, Personal dead reckoning system with shoe mounted inertial sensors. PhD thesis, 2008.

[6] B. Krach and P. Robertson, "Integration of foot-mounted inertial sensors into a Bayesian location estimation framework," 2008 5th Workshop on Positioning, Navigation and Communication, vol. 2008, pp. 55-61, Mar. 2008.

[7] A. Jimenez, F. Seco, J. Prieto, and J. Guevara, "A comparison of Pedestrian Dead-Reckoning algorithms using a low-cost MEMS IMU," in 2009 IEEE International Symposium on Intelligent Signal Processing, pp. 37-42, Ieee, Aug. 2009.

[8] S. J. Julier and J. K. Uhlmann, "A new extension of the kalman filter to nonlinear systems," pp. 182-193, 1997.

[9] E. Wan and R. Van Der Merwe, "The unscented kalman filter for nonlinear estimation," pp. 153 - 158, 2000.

[10] S. S. Haykin, Kalman filtering and neural networks. Wiley, 2001.

[11] A. Jimenez, F. Seco, J. Prieto, and J. Guevara, "Indoor Pedestrian Navigation using an INS/EKF framework for Yaw Drift Reduction and a Foot-mounted IMU," in WPNC 2010: 7th Workshop on Positioning, Navigation and Communication, vol. 10, 2010.

[12] M. H. Afzal, Use of Earth's Magnetic Field for Pedestrian Navigation. PhD thesis, University of Calgary, July 2011.

[13] R. Stirling, "Development of a Pedestrian Navigation System Using Shoe Mounted Sensors," Master's thesis, University of Alberta, 2004.

[14] L. Ojeda and J. Borenstein, "Non-GPS navigation with the personal dead-reckoning system," in SPIE Defense and Security Conference, Unmanned Systems Technology IX, Orlando, Florida, April 9-13, 2007, pp. 65610C-65610C-11, Spie, 2007.

[15] I. Skog, P. Handel, J. Nilsson, and J. Rantakokko, "Zero-velocity detection - an algorithm evaluation," Biomedical Engineering, IEEE Transactions on, vol. 57, pp. 2657 -2666, nov. 2010.

[16] F. J. Zampella, A. R. Jimnez, F. Seco, J. C. Prieto, and J. I. Guevara, "Simulation of Foot-Mounted IMU Signals for the Evaluation of PDR Algorithms," in International Conference on Indoor Positioning and Indoor Navigation (IPIN), 2011.

[17] M. Angermann, P. Robertson, T. Kemptner, and M. Khider, "A High Precision Reference Data Set for Pedestrian Navigation using FootMounted Inertial Sensors," in 2010 International Conference on Indoor Positioning and Indoor Navigation (IPIN), 15-17 September 2010, Zürich, Switzerland, no. September, p. 6, 2010.

[18] M. Afzal, V. Renaudin, and G. Lachapelle, "Magnetic field based heading estimation for pedestrian navigation environments," in Indoor Positioning and Indoor Navigation (IPIN), 2011 International Conference on, pp. $1-10$, sept. 2011.

[19] M. H. Afzal, V. Renaudin, and G. Lachapelle, "Use of earths magnetic field for mitigating gyroscope errors regardless of magnetic perturbation," Sensors, vol. 11, no. 12, pp. 11390-11414, 2011. 